\title{
Hitos innovadores en la oftalmología
}

\section{Innovative milestones in Ophthalmology}

Jaime Lozano-Alcázar*

Departamento de Dirección Médica, Fundación Hospital Nuestra Señora de la Luz, Ciudad de México, México

\begin{abstract}
Resumen
Todo médico es potencialmente un investigador. Grandes aportaciones al desarrollo de la medicina se han debido a hechos fortuitos. En la oftalmología, por ejemplo, el efecto de un eclipse de sol fue la idea inicial de la endofotocoagulación; un aparato dental dio lugar a la facoemulsificación; el comentario de un estudiante de medicina despertó la idea de los lentes intraoculares. Pero, como dijo Pasteur: "Dans les champs de l'observation, le hasard ne favorise que les esprits préparés" (en el campo de la observación, la fortuna solo favorece a los espíritus preparados). Se presentan aquí algunos personajes y sus principales aportaciones al desarrollo de la oftalmología, esperando que su ejemplo sirva para despertar o avivar el interés por la investigación.
\end{abstract}

Palabras clave: Historia de la oftalmología. Oftalmólogos innovadores. Avances en oftalmología.

\section{Abstract}

Every physician is potentially a researcher. Important contributions to medicine development have been due to fortuitous observations. In Ophthalmology, for example, the initial idea of endophotocoagulation was the effect of a solar eclipse; a dental device resulted in phacoemulsification; the comment of a medical student led to intraocular lenses. But, as Pasteur said: "Dans les champs de l'observation, le hasard ne favorise that esprits préparés" (in the fields of observation, chance favors only the prepared mind). Some authors and their contributions to the development of Ophthalmology are presented here, hoping that their example will serve to arouse or increase interest in research.

Key words: History of Ophthalmology. Innovative ophthalmologists. Advances in Ophthalmology.

Los formidables avances que han tenido en las últimas décadas el diagnóstico y terapéutica médica y quirúrgica en nuestra especialidad no hubieran podido llevarse a cabo sin las bases que se fueron forjando desde la más remota antigüedad, muchas veces por el conocimiento empírico, que hoy suele ser menospreciado.

La investigación de manera más formal se inició en la segunda mitad del siglo XIX. Veremos ahora algunas de las contribuciones más destacadas, con la intención no solo de recordar como anécdota histórica a los investigadores y sus aportaciones, sino más bien para que de alguna manera sirvan de estímulo a los investigadores de la actualidad. Cada médico, al menos en potencia, es un investigador.

Desde luego, el gran detonador para la consolidación de la oftalmología como especialidad fue la invención

\section{Correspondencia:}

*Jaime Lozano-Alcázar

Ezequiel Montes, 135

Col. Tabacalera

Fecha de recepción: 06-01-2020

Ciudad de México, México

E-mail: Drjaimelozano@gmail.com

DOI: 10.24875/RMO.M20000113
Disponible en internet: 01-07-2020 Rev Mex Oftalmol. 2020;94(4):178-181

www.rmo.com.mx (http://creativecommons.org/licenses/by-nc-nd/4.0/). 
del oftalmoscopio, y tanto para la cirugía de la especialidad como para la cirugía en general, la aplicación de la anestesia y la antisepsia.

En este tema no se puede soslayar la mención a los dos más grandes personajes del siglo antepasado para el desarrollo de la medicina. Uno, el gran Louis Pasteur (1822-1895), biólogo, microbiólogo y químico francés, reconocido por la vacunación, especialmente de la rabia y el ántrax, la esterilización de la leche y la cerveza por medio de la pasteurización, y por múltiples investigaciones sobre microbiología: su relación con enfermedades y su aplicación en la medicina clínica. Es conocido que puso fin a la teoría de la generación espontánea, hasta ese momento tan aceptada. En relación directa con nuestra especialidad descubrió la Pasteurella septica, causante de septicemia hemorrágica en las aves y que eventualmente puede causar conjuntivitis por contagio a los granjeros ${ }^{1}$. La otra gran figura es Joseph Lister (1827-1912), el cirujano inglés que aplicó a la cirugía los descubrimientos de Pasteur. Insistió en una cirugía estéril mediante la asepsia y antisepsia, e introdujo la esterilización del instrumental y del sitio de la herida quirúrgica por medio del ácido carbólico (fenol). Propuso que los mangos de los instrumentos quirúrgicos no fueran de material poroso. Pugnó también por el lavado de manos de los cirujanos antes y después de la intervención quirúrgica, como ya antes habían postulado Ignaz Semmelweis (1818-1865) en Suiza y Oliver Wendell Holmes (1809-1894) en EE.UU. En cuanto a la oftalmología, Lister investigó la musculatura del iris. Su principal auxiliar en investigaciones fue su esposa Agnes².

Friedrich Wilhelm Ernst Albrecht von Gräfe (1828-1870), como se puede ver, von Gräfe murió muy joven, con apenas 42 años, de tuberculosis pulmonar, pero dejó una huella memorable. Se le considera el más importante oftalmólogo del siglo XIX. Introdujo la iridectomía para el manejo del glaucoma; identificó la asincronía del abatimiento del párpado superior a la infraversión en los pacientes con orbitopatía tiroidea (signo de von Gräfe), y describió la oftalmoplejia progresiva externa. En 1855 fundó la revista Archiv für Ophthalmologie, que aún persiste, y en 1863, la Deutsche Ophthalmologische Gesellschaft. Diseñó el famoso cuchillete con su nombre, que aún se usaba en cirugía de catarata a mediados del siglo pasado. Su principal cualidad era aplicar asertivamente las aportaciones de otros, en particular el oftalmoscopio de Helmholtz. Así, fue el pionero en describir, interpretar y dibujar el fondo de ojo y sus alteraciones; describió la asociación de retinosis pigmentaria y sordera en el síndrome de Usher, el papiledema en cuatro pacientes con tumor cerebral; fue el primero en llevar un registro gráfico de las campimetrías patológicas; inició la exploración de tumores oculares mediante la transiluminación, y también impulsó el uso del tonómetro ${ }^{3}$.

Louis de Wecker (1832-1906), de origen francés, nacido en Alemania, tuvo en París una exitosa clínica para enfermos de los ojos. Fue el inventor de la cirugía filtrante para el glaucoma crónico con su esclerotomía anterior $^{4}$, que presentó en 1856, 2 años después de que von Gräfe diera a conocer su iridectomía. Más tarde, en 1900, fue publicada la cirugía filtrante interna, llamada ciclodiálisis, y en 1932, la ciclodestrucción. Más de 150 años después de las aportaciones de von Gräfe y Wecker, y otros, los cuatro métodos: solución del bloqueo pupilar, filtración externa, filtración interna y disminución de la producción del acuoso son aún las técnicas básicas en el manejo quirúrgico del glaucoma. La trabeculectomía, publicada por Cairns en 1968, se convirtió pronto en la técnica de referencia en cuanto a cirugías filtrantes, aunque en la misma época surgieron cirugías filtrantes no penetrantes que no tuvieron mayor aceptación. Poco después, en 1969, Molteno presentó el primer dispositivo filtrante efectivo. Desde 1995, la mayoría de nuevas técnicas quirúrgicas para el glaucoma preconizan el uso de dispositivos implantables de filtración externa o interna como el iStent, el ExPRESS, el SOLX ${ }^{5-7}$ y otros, agrupados con el término MIGS.

Manuel Uribe y Troncoso (1867-1959), nacido en Toluca, Estado de México. Fue médico del Hospital de la Luz. En 1893 fue firmante del acta constitutiva de la Sociedad Oftalmológica, hoy la Sociedad Mexicana de Oftalmología, la primera de médicos especialistas en América Latina, y fundó, en 1898, los Anales de Oftalmología, la actual Revista Mexicana de Oftalmología, la publicación más antigua de la especialidad en español. En 1900 fue el invitado de honor de la American Medical Association al congreso celebrado en Atlantic City; en 1910 fue delegado de México al Congreso Internacional de Higiene en Berlín, y en 1921, al Congreso Internacional de Higiene Escolar en París, pues fue Jefe del Servicio de Higiene Escolar e Inspector General Médico de las Escuelas del Distrito Federal y fundador de los Anales de Higiene Escolar, fundador del Departamento de Psicopedagogía y Médico Escolar, dependencia de la Secretaría de Educación Pública desde 1908. Fue catedrático de la Facultad de Medicina de la Universidad Nacional de México hasta 1916, cuando por razones políticas se autoexilió, radicándose en Nueva York. Ese mismo año 
se le nombró Profesor de Oftalmología en la New York Graduate Medical School and Hospital (en 1926 profesor titular) y fue fundador de la Sociedad Médica Hispano-Americana. En 1932 ingresó en el College of Physicians and Surgeons de la Columbia University and Presbyterian Hospital. Más tarde, la Universidad de Nueva York le dio el nombramiento de Profesor Extraordinario de Oftalmología. En 1933 asistió como Delegado de la Columbia University al XIX Congreso Internacional de Oftalmología en Madrid, y fue invitado después a dar conferencias en Valencia, Barcelona, París, Oxford, el Moorfields Eye Hospital de Londres, entre otros. Fue miembro de la Academia de Medicina de Nueva York, de la Sociedad Oftalmológica de Francia, del Laboratorio del Instituto Oftalmológico de la Universidad de Columbia, de la Sociedad Oftalmológica de Bélgica, miembro honorario de la Sociedad de Oftalmología Hispano-Americana de Madrid, redactor del American Journal of Ophthalmology. En 1942 recibió el Premio Anual de Investigación de la American Medical Association. Al año siguiente, la Secretaría de la Asistencia Pública de México le otorgó una condecoración, y el 15 de mayo de ese mismo año de 1943, por decreto presidencial de don Manuel Ávila Camacho, se le designó Miembro Fundador del Colegio Nacional. Obviamente, todas estas distinciones se debieron a sus eficaces investigaciones en el campo de la Oftalmología, se le considera el padre de la gonioscopia clínica, destacan entre otras la creación de un gonioscopio monocular autoiluminado, un microscopio corneal binocular, un modelo de ojo para demostración de ametropías. Escribió más de 170 artículos científicos y los libros Internal Diseases of the Eye y Atlas of Gonioscopy ${ }^{8}$. Es el oftalmólogo mexicano más reconocido a nivel internacional.

Jules Gonin (1870-1935), oftalmólogo suizo. En los inicios del siglo XX, tras una intensa y productiva investigación sobre la fisiopatogenia del desprendimiento de retina y el significado de los desgarros, ideó la ignipuntura, el primer método eficaz para resolver el desprendimiento de retina al cauterizar el tejido para crear un foco inflamatorio cuya cicatrización causa la adherencia. Presentó su método en 1919 a la Sociedad Oftalmológica Suiza, pero pasaron varios años para que se le diera crédito, a lo que contribuyeron testimonios de Arruga, Amsler y Veil ${ }^{9,10}$. Dado el alto riesgo de complicaciones, la técnica fue sustituida, años después, por la fotocoagulación.

Sir William Stewart Duke-Elder (1898-1978), oftalmólogo inglés, incansable investigador bibliográfico. Entre otros muchos escritos publicó dos obras monumentales el Text Book of Ophthalmology de 7 tomos y el System of Ophthalmology, 15 volúmenes en 19 tomos. Destacó como director de investigación en el Instituto de Oftalmología de Londres ${ }^{11}$.

Hans Goldmann (1899-1991), nació en AustriaHungría y estudió medicina en Praga. Después de haber sido asistente de Anton Elschnig, hizo aportaciones notables que no pierden actualidad. Gracias a él tenemos la lámpara de hendidura tal y como ahora la conocemos, el perímetro, el lente de tres espejos, el tonómetro de aplanación, el adaptómetro, el fluorofotómetro y el colorímetro, por supuesto, todos llamados de Goldmann. Recibió la Medalla Gonin y doctorados honoris causa por las universidades de Uppsala, Estrasburgo y Ginebra ${ }^{12}$.

Sir Nicholas Harold Lloyd Ridley (1906-2001). Este cirujano inglés fue el inventor del lente intraocular para la rehabilitación de la afaquia. El comentario de un estudiante de medicina que lo vio extraer una catarata y le preguntó por qué no sustituía el lente lo inspiró para crear el lente intraocular, seleccionando como material el metilmetacrilato polimerizado, pues había observado que cuerpos extraños intraoculares de ese material se mantenían inertes en pilotos de la Real Fuerza Aérea; las cabinas de los Spitfire eran de ese material. En el St. Thomas Hospital de Londres, el 29 de noviembre de 1949, hizo el primer implante. El ojo quedó con miopización de 14 dioptrías, mejoró sus cálculos de tal manera que el segundo ojo quedó solamente con 1 dioptría negativa ${ }^{13}$. Durante la II Guerra Mundial, mientras servía en el ejército inglés en oriente hizo estudios de la oncocercosis, la acción en los ojos del veneno de la cobra escupidora y la ambliopía nutricional ${ }^{14}$.

Gerhard Rudolph Edmund Meyer-Schwickerath (1920-1992), es un oftalmólogo alemán. Se le considera el padre de la endofotocoagulación de la retina y de las cirugías oculares que de ahí se derivaron. Tuvo la oportunidad de examinar a varios pacientes que habían tenido quemaduras en la retina por observar el eclipse solar del 9 de julio de 1945, y en 1946-1947 llegó a la conclusión de que las cicatrices resultantes eran por un efecto similar al de la diatermia de superficie que ya se usaba para la cirugía del desprendimiento de retina. Aplicando los postulados de Gonin, dedujo que con cicatrices provocadas por la luz del sol en sitios precisos podía detener el progreso de los desprendimientos de retina e inició los primeros experimentos con fotocoagulación. En 1949 obtuvo el primer tratamiento exitoso en la azotea de la Clínica de Oftalmología de la Universidad de HamburgoEppendorf, con un aparato diseñado por él que mediante un sistema de lentes de aumento y espejos permitía 
enfocar la luz del sol al interior del ojo. Desafortunadamente, los días nublados impedían el tratamiento, así que, hacia 1950 desarrolló, con la compañía Zeiss, la «lámpara de descarga de gas xenón de alta presión» que, obviamente, ya no dependía del sol. Más tarde, los fotocoaguladores de xenón fueron sustituidos por los que usan energía láser, como todos sabemos ${ }^{15,16}$.

Charles D. Kelman (1930-2004). Hizo su residencia en el Wills Eye Hospital de Filadelfia, y trabajó en el Manhattan Eye, Ear and Throat Hospital en Nueva York. En 1992, recibió la Medalla Nacional de Tecnología del Presidente George H. W. Bush y fue introducido al National Inventors Hall of Fame, en Akron, Ohio, por su invento del facoemulsificador y la descripción de la técnica quirúrgica. Según él mismo decía, la facoemulsificqación le fue inspirada por el instrumento de ultrasonido que usaba su dentista ${ }^{17}$.

No quiero terminar sin mencionar que además del Dr. Uribe y Troncoso otros oftalmólogos mexicanos han hecho aportaciones importantes que han dejado documentadas, desafortunadamente no es fácil encontrar las citas bibliográficas, haciendo un breve ejercicio de memoria recuerdo algunos ejemplos en el ámbito quirúrgico

El Dr. Arcadio Chacón Mendoza, en el Boletín del Hospital Oftalmológico de Nuestra Señora de la Luz, tomo XV, año XXIII, n. ${ }^{\circ} 46$, segunda época, julio a septiembre de 1962, páginas 125 a 129, describe por primera vez, según el escrito mismo señala, la maniobra que lleva su nombre para manejo de la dacrioestenosis congénita y que le valió un amplio reconocimiento internacional; en las paredes de su consultorio en Mexicali, B.C., se encontraban diplomas de sociedades oftalmológicas europeas y del continente americano en que lo nombraban miembro correspondiente.

El Dr. Antonio Torres Estrada, entre otras, describió una técnica para manejo del ectropión del punto lagrimal, que se llamó «punto de Torres Estrada», que aún es totalmente vigente.

El Dr. Luis Sánchez Bulnes, entre otros notables logros, tuvo el de idear la técnica de las «resecciones radiadas» para hacer un «cincho» natural al globo ocular para manejo del desprendimiento de retina.

\section{Conclusión}

Para terminar, quisiera dejar la idea de que potencialmente todo oftalmólogo puede hacer una investigación que lleve a una aportación valiosa. Como hemos visto, la idea inicial de la endofotocoagulación fue el efecto de un eclipse de sol, un aparato dental dio lugar a la facoemulsificación, la observación de un estudiante de medicina, a los lentes intraoculares, y cuántas otras causas fortuitas habrá en la historia, lo importante es saber aprovechar la idea inicial, para eso es necesario lo que dice Pasteur: «Dans les champs de l'observation, le hasard ne favorise que les esprits préparés» (en el campo de la observación la fortuna solo favorece a los espíritus preparados).

\section{Bibliografía}

1. Duke-Elder S. Diseases of the Outer Eye. Vol. VIII, part 1. En: Duke-Elder S, editor. System of Ophthalmology. London: H. Kimpton; 1965. p. 210.

2. Duke-Elder S, Wylar KC. The Anatomy of the Visual System. Vol. II. En: Duke-Elder S, editor: System of Ophthalmology. London: H. Kimpton; 1961. p. 178-9.

3. Duke-Elder S. The Foundations of Ophthalmology. Vol. VII. En: Duke-Elder S, editor: System of Ophthalmology. London: H. Kimpton; 1962. p. 232-3.

4. Duke-Elder S. Diseases of the Lens and Vitreous; Glaucoma and Hypotony. Vol. XI. En: Duke-Elder S, editor. System of Ophthalmology. London: H. Kimpton; 1969. p. 228.

5. Razeghinejad MR, Spaeth GL. A History of the Surgical Management of Glaucoma. Optom Vis Sci. 2011;88(1):E39-47.

6. Molteno AC. New Implant for Drainage in Glaucoma. Br J Ophthalmol. 1969;53:606-15.

7. Cairns JE. Trabeculectomy. Premilinary Report of a New Method. Am J Ophthalmol. 1968;66(4):673-9.

8. http://colnal.mx/members/manuel-uribe-troncoso. Último acceso: 20 de febrero de 2018

9. Duke-Elder S, Dobree JH. The Retina. Vol. X. En: Duke-Elder S, editor. System of Ophthalmology. London: H. Kimpton; 1967. p. 771-3.

10. Hernández-DaMota SE. Historia de la cirugía del desprendimiento de retina hasta Gonin. Rev Mex Oftalmol. 2008;82(5):331-4.

11. https://en.wikipedia.org/wiki/Stewart_Duke-Elder. Último acceso: 20 de marzo de 2018.

12. https://en.wikipedia.org/wiki/Hans_Goldmann. Último acceso: 20 de marzo de 2018.

13. Ridley $\mathrm{H}$. The Cure of Aphakia. The History of Lens Implantation. Section 3. En: Rosen ES, Hainning WM, Arnott FJ, editors. Intraocular Lens Implantation. St Louis: The C. V. Mosby Co. 1984. p. 37-42.

14. https://en.wikipedia.org/wiki/Harold_Ridley_(ophthalmologist. Último acceso: 21 de febrero de 2018.

15. https://en.wikipedia.org/wiki/Gerhard_Meyer-Schwickerath. Último acceso: 21 de febrero de 2018.

16. Duke-Elder S, Mac Fane A, Injuries. Vol, XIV, n.ำ 2. En: Duke-Elder S, editor. System of Ophthalmology. London: H. Kimpton; 1972. p. 897.

17. https://en.wikipedia.org/wiki/Charles_Kelman. Último acceso: 21 de febrero de 2018. 